\title{
Graticule incorporated into an ophthalmoscope for the clinical evaluation of the cup/disc ratio
}

\author{
J. H. ROMANO
}

SUMmARY A graticule incorporated into a newly developed ophthalmoscope, and designed to be projected onto the fundus, is presented as an aid to the visual evaluation of the cup/disc ratio.

One of the best established methods of screening for and of detecting the early signs of open-angle glaucoma is the examination of the optic disc. Clinically this includes an evaluation of the degree of excavation and the degree of pallor of the disc, along with other morphological changes. Of these the degree of excavation has proved to be the most reliable guide, particularly in screening for glaucoma by general practitioners, ophthalmic opticians, and other nonspecialist ophthalmological workers.

Any change in the degree of excavation is of course of the greatest significance in the follow-up of established glaucoma cases or in the diagnosis of doubtful cases.

The concept of the cup/disc ratio, introduced by Armaly, ${ }^{1}$ has proved despite its shortcomings to be a clinically useful method of describing the changes observed. Other terms used to describe cupping of the discs, such as 'early cupping,' 'moderate or severe cupping,' 'pathological cupping,' etc., are nondescriptive, ambiguous, and largely meaningless. The cup/disc ratio attempts to give a numerical estimate of the degree of excavation, and this can be transmitted in correspondence, over the telephone, or whenever photography of the optics discs is not available.

\section{Description of instrument}

In this ophthalmoscope (Fig. 1) one has endeavoured to facilitate evaluation of the cup/disc ratio by providing a newly designed graticule which can be projected on to the optic disc (Fig. 2). The graticule consists of an outer circle which will be found to coincide with the edge of the optic disc in most cases. This is considered as unity. The concentric circles are calculated to represent a cup/disc ratio of 0.5 for the middle circle and 0.25 for the inner circle. The notches between the outer and the middle circle represent a cup/disc ratio of $0 \cdot 75$.

Correspondence to Mr J. H. Romano, FRCS, 96 Harley Street. London W1N 1AF.

\section{Instructions for use}

The use of a dilated or semidilated pupil facilitates the examination. It is recommended that the disc be first examined with a normal ophthalmoscope beam. The graticule is then inserted into the pathway of the beam and is first projected on to the retina adjacent to the disc and brought into the sharpest possible focus by adjusting the ophthalmoscope lenses before being

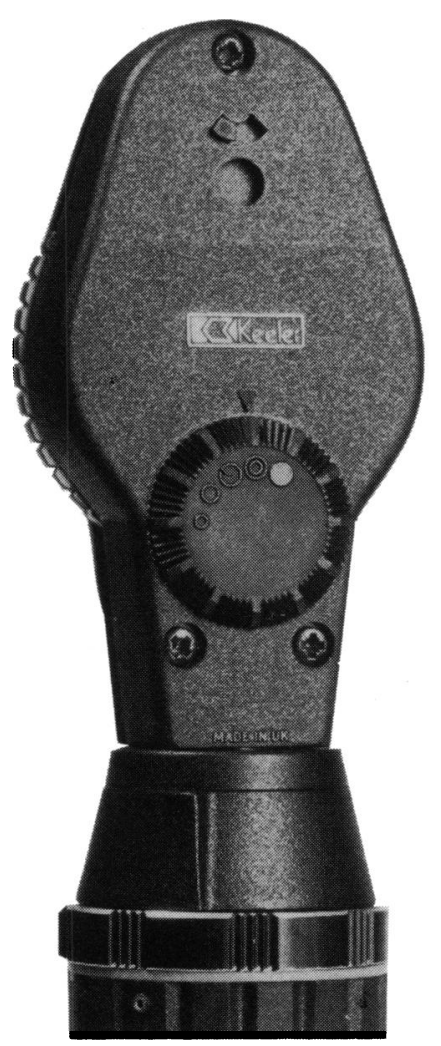

Fig. 1 The ophthalmoscope.

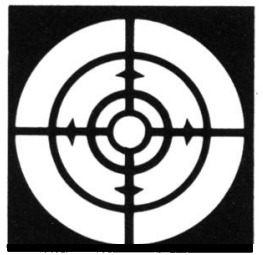

Fig. 2 The graticule. 
moved onto the disc. Visualisation of the graticule is always easier and better defined on the retina than on the optic disc. The graticule is swung on to the optic disc, and adjustment of the focusing lenses is repeated until it shows up most clearly. The graticule should be swung away from the disc while this is still being examined and then swung back if necessary several times until an opinion is obtained as to which circle most closely approximates to the optic cup. The corresponding $\mathrm{C} / \mathrm{D}$ ratio is then read off.
If the disc presents a peripapillary rim or halo, one must be careful not to project the outer circle of the graticule on to the edge of the rim but on to the edge of the disc proper.

The graticule was developed in collaboration with Keeler Instruments Ltd., of London, and my sincere thanks are extended to $\mathrm{Mr}$ Clive Burrows for his painstaking efforts on its behalf.

Reference

1 Armaly A. The cup/disc ratio. Arch Ophthalmol 1969; 82: 191-6. 